\title{
Personalized implementation of video telehealth for rural veterans (PIVOT-R)
}

\author{
Stephanie C. Day ${ }^{1,2,3}$, Giselle Day ${ }^{2}$, Michele Keller ${ }^{4}$, Hilary Touchett ${ }^{1}$, Amber B. Amspoker ${ }^{1,3}$, \\ Lindsey Martin ${ }^{1,3 *}$, Jan A. Lindsay ${ }^{1,2,3}$ \\ ${ }^{1}$ Houston VA HSR\&D Center for Innovations in Quality, Effectiveness and Safety, Michael E. DeBakey Veterans Affairs Medical Center, Houston, \\ TX, USA; ${ }^{2}$ VA South Central Mental Illness Research, Education and Clinical Center, Houston, TX, USA; ${ }^{3}$ Baylor College of Medicine, Houston, \\ TX, USA; ${ }^{4}$ Veterans Affairs Medical Center-Fort Harrison, Fort Harrison, MT, USA \\ Contributions: (I) Conception and design: SC Day, JA Lindsay; (II) Administrative support: G Day; (III) Provision of study materials or patients: M \\ Keller, JA Lindsay, SC Day; (IV) Collection and assembly of data: L Martin, AB Amspoker, G Day; (V) Data analysis and interpretation: L Martin, \\ AB Amspoker; (VI) Manuscript writing: All authors; (VII) Final approval of manuscript: All authors. \\ Correspondence to: Stephanie C. Day. MEDVAMC 152, 2002 Holcombe Blvd., Houston, TX 77030, USA. Email: stephanie.day2@va.gov.
}

Background: A national shortage of mental health $(\mathrm{MH})$ professionals leaves more than $90 \%$ of rural individuals without adequate access to services each year, troubling because $33 \%$ of Veterans Health Administration (VHA) enrollees live in rural areas and rural Veterans have a greater risk of suicide than urban Veterans. Additional barriers such as travel distance and cost, stigma and extreme weather or geography add to challenges of rural Veterans seeking treatment. Although the VHA has addressed this disparity by providing telemental health services, provision of services via traditional hub-and-spoke and/or establishment of regional centers has not fully addressed barriers or resource limitations. Video telehealth to home (VTH) has assisted in better addressing geographic, attitudinal and systematic barriers to in-person care; however, its uptake and implementation have been problematic. This article describes the Personalized Implementation of Video Telehealth for Rural Veterans (PIVOT-R) approach, developed in response to the unique needs of rural veterans.

Methods: We developed PIVOT, a flexible implementation strategy that is adaptive to site-specific contexts and different digital innovations and relies on a collaborative relationship between external facilitators, internal facilitators and clinical champions. We used formative evaluation (FE) to gather ongoing information about our quality improvement (QI) implementation approach of VTH. Our FE of PIVOT at rural sites provided insight into adaptations to improve rural implementation. This led to development of PIVOT-R, which explicitly focuses on rural implementation. PIVOT-R, developed from provider and patient feedback plus lessons learned during implementation, focuses on rurality as an important diversity factor and addresses relationship building, engaging the site, assessing context and infrastructure and balancing national expectations with site-level goals. During fiscal year 2018 we partnered with a VHA healthcare system in a Western mountain state to pilot the PIVOT-R approach, again using FE which included quantitative and qualitative data collection to evaluate its impact.

Results: PIVOT-R effectively increased uptake of VTH for MH care at the healthcare system evaluated. In fiscal year 2019 the percentage of Veterans receiving MH care via VTH at the site was 10 times greater than in fiscal year 2018, matching the mean VHA nationwide percentage and increasing by $43.24 \%$ by the end of 2019. Veteran feedback supported a positive experience by users.

Conclusions: Inclusion of a comprehensive assessment of the rural system, including infrastructure and resources, greatly improves understanding of a system's specific needs and enables a tailored approach

\footnotetext{
*Now affiliated with the National Institute of Environmental Health Sciences, Durham, NC, USA
} 
targeting relevant barriers. Our FE suggests the potential of PIVOT-R to increase VTH uptake at other rural locations and reinforces the value of telehealth technology as an important resource for rural sites.

Keywords: Veterans; centers; rural health; mental health services; telemedicine; implementation; health plan

Received: 18 January 2020; Accepted: 09 March 2020; Published: 20 April 2021.

doi: $10.21037 /$ mhealth.2020.03.02

View this article at: http://dx.doi.org/10.21037/mhealth.2020.03.02

\section{Introduction}

Nationally, the mental health $(\mathrm{MH})$ professional shortage leaves more than $90 \%$ of rural individuals without adequate access to services each year $(1,2)$. Approximately $65 \%$ of rural counties are without a psychiatrist, and $47 \%$ of rural counties lack a psychologist, severely limiting the availability of $\mathrm{MH}$ care in rural areas $(3,4)$. Beyond access disparities, additional barriers such as travel distance and cost, stigma of seeking $\mathrm{MH}$ services, and extreme weather or geography create challenges to providing in-person $\mathrm{MH}$ care to rural populations $(5,6)$.

Among rural Veterans, $\mathrm{MH}$ care is a critical concern, as one in five post-deployment Veterans met criteria for an MH diagnosis; (6) and 33\% of Veterans Health Administration (VHA) enrollees live in rural areas (5). Veterans in rural areas report poorer $\mathrm{MH}$, have more severe MH diagnoses, and are more likely to die by suicide than urban veterans $(7,8)$. The suicide rate among veterans is 1.5 times higher compared to non-veterans, with the highest concentrations in western and rural states $(9,10)$.

Telemental health is one solution to address barriers and improve access to $\mathrm{MH}$ care, particularly for patients in rural areas. Early telehealth in VHA used a hub-and-spoke model to increase access to specialty $\mathrm{MH}$ care by connecting a provider at a medical center to a patient at an affiliated community clinic. A network approach was later employed to address gaps in coverage, connecting a provider and patient at different community clinics within the same healthcare network. To keep up with the telehealth demand, VHA also implemented telemental health resource centers, which provide $\mathrm{MH}$ care parallel to the hub-and-spoke or network models. Resource centers cluster $\mathrm{MH}$ providers at one location where they deliver care to patients in community clinics, not otherwise affiliated with the resource center, oftentimes in a geographically distant location (i.e., different state). Although the hub-spoke, network, and resource center models improve access to evidence-based MH care, they do not fully address barriers or resource limitations. Patients still travel to a facility to attend telehealth sessions, resulting in time and travel costs, and both the patient and provider require private rooms with telehealth equipment at their respective locations, which can further exacerbate issues of limited space. While hubspoke and network models enable patients to be seen either in-person or via telehealth, based upon patient preference and clinical indications, resource centers do not allow inperson appointments (see Table 1: models of telehealth).

Recent technological advances have resulted in video telehealth to home (VTH), a secure and encrypted web-based video conferencing format, which enables a patient to connect with a provider from any private and convenient location (i.e., home, work, local library, or parked car), using an internet or cellular connection. Since VTH was first approved in 2014 by VHA, it has revised national expectations for VTH, initially encouraging and later requiring provider adoption of VTH delivery. VTH is equivalent to in-person visits regarding clinical effectiveness (11) and patient satisfaction outcomes (12-14). VTH delivery leads to fewer cancellations because it better addresses geographic, attitudinal, and systematic barriers to in-person care (13). Furthermore, VTH for MH care is more cost effective, as it is currently offered at no cost to veterans, is accessible through most veterans' personal devices, and reduces Veterans Affairs (VA) travel pay costs (i.e., mileage reimbursement) $(15,16)$ for eligible veterans $(12,13,15)$. In 2019 the VA Office of Connected Care reported that $90 \%$ and $88 \%$ of veterans were satisfied or very satisfied with telehealth services and telehealth home/mobile apps respectively (17). VHA providers who adopted VTH for $\mathrm{MH}$ reported overall positive experiences and noted benefits to their patients $(18,19)$. Although VTH addresses many barriers to care, a combination of telehealth approaches may be needed to meet the clinical needs and preferences of rural patients. For example, current interpretations of the Ryan Haight Act state that a provider must meet with a patient at a Drug Enforcement Administration-registered clinic before writing a prescription for a controlled substance (20). This 
Table 1 Models of telehealth

\begin{tabular}{|c|c|c|c|}
\hline Model & Description & Pros & Cons \\
\hline \multirow{2}{*}{ Hub-and-spoke } & & $\begin{array}{l}\text { Provider and patient located in } \\
\text { same general area }\end{array}$ & $\begin{array}{l}\text { Requires telehealth staff at patient } \\
\text { location }\end{array}$ \\
\hline & & Possibility of in-person visits & \\
\hline \multirow[t]{4}{*}{ Network } & $\begin{array}{l}\text { Provider at medical center or } \\
\text { community clinic }\end{array}$ & $\begin{array}{l}\text { Addresses some barriers to } \mathrm{MH} \\
\text { care }\end{array}$ & Patient still travels to clinic \\
\hline & $\begin{array}{l}\text { Patient at medical center or } \\
\text { community clinic in same network }\end{array}$ & $\begin{array}{l}\text { More reliable connectivity due to } \\
\text { "wired" internet }\end{array}$ & $\begin{array}{l}\text { Requires room and equipment at both } \\
\text { locations }\end{array}$ \\
\hline & & $\begin{array}{l}\text { Provider and patient located in } \\
\text { same general area }\end{array}$ & \\
\hline & & Possibility of in-person visits & \\
\hline \multirow[t]{3}{*}{ Resource center } & $\begin{array}{l}\text { Provider at telehealth silo (only } \\
\text { doing telehealth) }\end{array}$ & $\begin{array}{l}\text { Addresses some barriers to } \mathrm{MH} \\
\text { care }\end{array}$ & Patient still travels to clinic \\
\hline & Patient at community clinic & $\begin{array}{l}\text { More reliable connectivity due to } \\
\text { "wired" internet }\end{array}$ & $\begin{array}{l}\text { Requires room and equipment at both } \\
\text { locations }\end{array}$ \\
\hline & $\begin{array}{l}\text { Provider and patient are } \\
\text { geographically distant }\end{array}$ & $\begin{array}{l}\text { Providers develop expertise in } \\
\text { telehealth delivery }\end{array}$ & $\begin{array}{l}\text { Requires telehealth staff at patient } \\
\text { location }\end{array}$ \\
\hline \multirow{4}{*}{$\begin{array}{l}\text { Video telehealth } \\
\text { to home (VTH) }\end{array}$} & & $\begin{array}{l}\text { Patients can use own or VA-issued } \\
\text { device }\end{array}$ & $\begin{array}{l}\text { Challenges with connectivity in highly } \\
\text { rural or mountainous areas }\end{array}$ \\
\hline & & $\begin{array}{l}\text { Can easily combine in-person and } \\
\text { VTH visits }\end{array}$ & \\
\hline & & $\begin{array}{l}\text { Patients can connect when in } \\
\text { another state }\end{array}$ & \\
\hline & & $\begin{array}{l}\text { Ability to see patient's home } \\
\text { environment }\end{array}$ & \\
\hline
\end{tabular}

$\mathrm{MH}$, mental health.

requirement means that providers may need to combine delivery modalities to ensure adherence to best practices and national guidelines.
Despite expectations within VHA and the benefits of VTH for MH care, uptake of this modality has been limited. System complexities, provider concerns about the 
impact of remote contact on patient-provider relationship, clinical workflow challenges, and logistical issues (e.g., scheduling and technical difficulties) create unique obstacles to implementing VTH. Increasing awareness of these difficulties led us to develop Personalized Implementation for Video Telehealth (PIVOT), a comprehensive approach to implementation that identifies and addresses site-specific challenges of VTH care $(21,22)$. Prior work shows that the PIVOT approach dramatically improved VTH adoption in VA medical centers where implemented $(21,22)$. PIVOT sites demonstrated improved rates of unique Veterans using VTH and total MH encounters via VTH $(21,22)$, increased numbers of providers offering VTH, and increased numbers of community and specialty $\mathrm{MH}$ clinics offering VTH as compared to national VHA counterparts without PIVOT intervention (18). Despite these successes, the PIVOT approach incompletely addresses the unique needs of sites and providers that serve rural Veterans. Here, we describe Personalized Implementation of Video Telehealth for Rural Veterans (PIVOT-R), an extension of the PIVOT strategy that incorporates rural considerations to create an approach for personalized implementation of VTH in rural settings.

\section{Methods}

\section{PIVOT}

PIVOT is a flexible strategy, grounded in implementation science, that is adaptive to site- specific contexts and different digital innovations. We developed PIVOT because technology-based innovations are notoriously difficult to implement, with national directives and leadership expectations rarely sufficient to improve uptake of innovations $(23,24)$. We used formative evaluation (FE) to continually assess the implementation process and VTH innovation, offering ongoing feedback and adjusting our implementation efforts to address site specific barriers (25). PIVOT is intended to work at the site level to support VTH expansion and national telehealth mandates. PIVOT is focused on capacity-building at the local level, working to help all providers integrate VTH delivery into their clinical practice rather than relying upon a few dedicated VTH providers.

The PIVOT approach begins with a site visit where external facilitators meet with medical center leadership and stakeholders (i.e., MH leadership, facility telehealth coordinator, IT leadership) to review VHA's national VTH goals, identify internal facilitator(s), and discuss where to initiate implementation (18). As PIVOT proceeds, the external and internal facilitators collaborate to advance and adapt implementation efforts.

PIVOT relies on a collaborative relationship between external facilitators, internal facilitators, and clinical champions to advance implementation efforts. External facilitators are individuals located outside the implementation site who act as change agents, guiding the implementation process and participating in collaborative problem solving $(9,26)$. Our external facilitators are health science researchers and clinicians with implementation facilitation training (26) and VTH expertise. External facilitators educate and update site leadership, train and troubleshoot with providers, and stay informed about continually evolving guidelines and best practices. Internal facilitators are designated staff at the implementation site trained to be the local VTH point of contact and communicate with on-site stakeholders. Clinical champions are providers in specialty $\mathrm{MH}$ or community clinics who champion VTH through adoption and endorsement of VTH with colleagues and patients. Although PIVOT flexibly adapts to each site, our experience using PIVOT to implement VTH in rural settings revealed the necessity of a rural-specific approach that emphasizes important considerations that are unique to rural sites, providers, and patients.

\section{PIVOT evaluation}

The QI, FE approach to evaluate PIVOT included feedback from internal facilitators (i.e., structured interviews, ongoing feedback from weekly or bi-weekly implementation meetings, communication from emails or instant messages), providers (i.e., direct feedback during site visits, communication from emails or instant messages), and patients (i.e., structured interviews) as well as quantitative data about VTH utilization obtained from the VHA Support Service Center Capital Assets Databases. We engaged in a continuous feedback loop to improve implementation, offering relevant information to internal facilitators and on-site stakeholders.

Our experience using PIVOT in rural settings highlighted how VTH can mean the difference between patients receiving care and having no care available. Although some rural communities have $\mathrm{MH}$ care available or are in proximity to an urban area with $\mathrm{MH}$ resources, many rural areas completely lack any $\mathrm{MH}$ providers. The 
Table 2 Veteran feedback-benefits of VTH

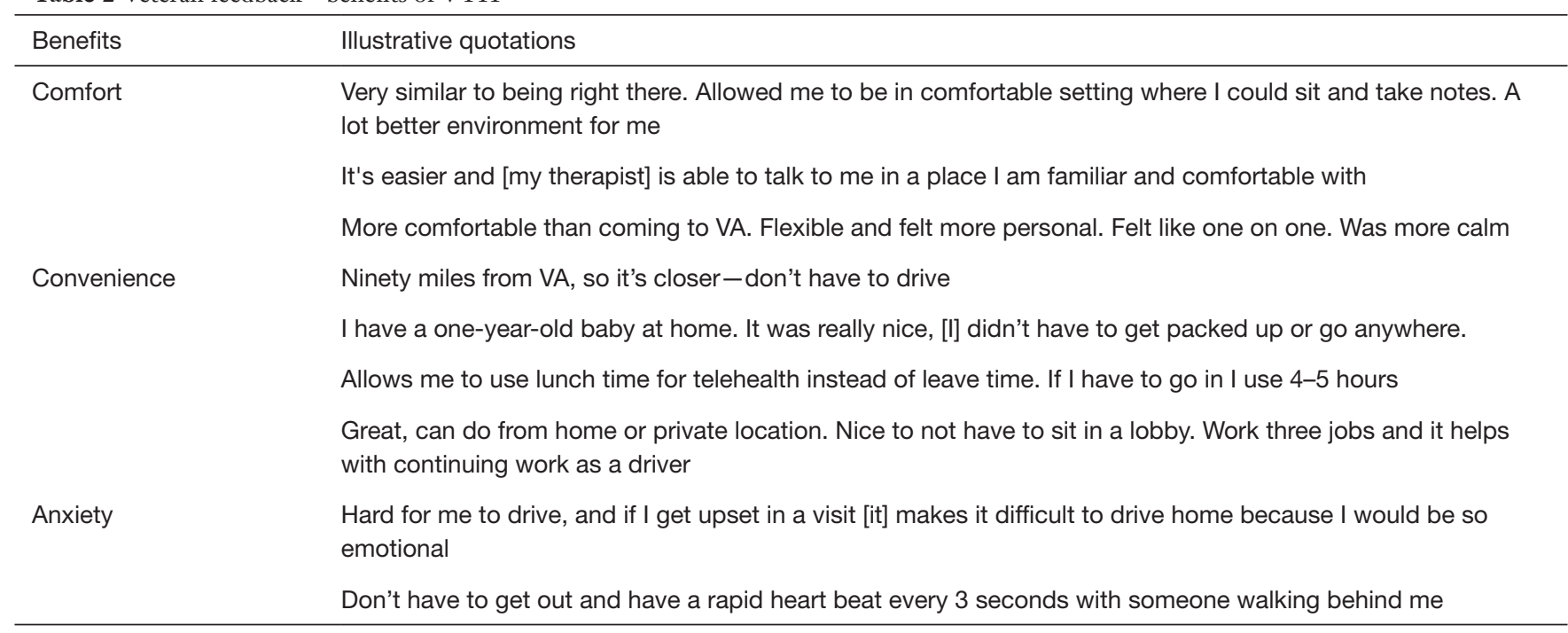

VTH, video telehealth to home.

awareness of the MH landscape often found in rural areas reinforced the importance of focusing on rurality in our implementation approach. Our PIVOT evaluation at rural sites provided insight into helpful adaptations to improve rural implementation. Rural patient and provider feedback highlighted oversights, blind spots, and implicit assumptions embedded in the PIVOT framework. We used these lessons learned in the development of PIVOT-R, an approach that explicitly focuses on rural implementation.

\section{Internal facilitator and provider feedback}

Internal facilitators recognized how limited resources, unclear expectations, and different provider perspectives and values can impact VTH uptake at rural sites. Internal facilitators described what they considered to be the unmatched dedication and work ethic of rural staff and providers while explaining that local patient stories illustrating how VTH improves access to $\mathrm{MH}$ care or quality of life are more persuasive than traditional motivators such as equivalent work credit or opportunities to telework. Using VTH marketing and messaging that reflects this aspect of the rural context may improve motivation for rural providers to incorporate VTH into their clinical practice.

Rural providers who used VTH were overwhelmingly positive about its ability to meet the needs of rural patients. Although they acknowledged that logistics (i.e., scheduling) and technology can be problematic, providers denied that either is prohibitive or significantly impacted their ability to build rapport with patients. Instead, many providers identified limited time to complete VTH training and provider reluctance to use VTH as the primary barriers to adoption. Providers consistently stressed the importance of targeted marketing for both providers and patients that emphasizes the specific challenges faced in rural communities. For example, providers discussed the power of including images and barriers/benefits of VTH that reflect rural communities.

\section{Veteran feedback}

Rural veterans who received care via VTH reported overall satisfaction despite occasional problems with technology. They discussed how VTH saved them travel time and cost, minimized time away from work or family responsibilities, and even improved their perceptions of the VA (see Table 2 for additional veteran voices). Furthermore, in rural areas with limited access to specialty MH care, VTH enabled veterans to engage in weekly therapy, often recommended for evidence-based practices (EBPs), with trained providers sometimes hundreds of miles away.

\section{PIVOT-R}

PIVOT-R, a strategy that focuses on rurality as an important diversity factor, was developed from provider and patient feedback, as well as lessons learned during implementation 
of VTH at rural sites. Rural patients and their providers identified specific barriers when implementing VTH and discussed the impact of rurality on uptake of technologybased innovations. PIVOT-R was adapted to prioritize and address rurality at the site, provider, and patient level.

\section{Rurality as a cultural factor}

Rurality is an important but sometimes overlooked aspect of diversity among veterans. Rural areas, the people who reside in them, and providers who work there are often perceived as homogeneous and may evoke stereotyped images of farmland or sparsely populated mountainsides, despite significant variability in rural geography and communities. Components of rurality (i.e., rural identity, cultural values, traditions, and perceptions of help seeking or $\mathrm{MH}$ care) can vary greatly by geographic region, proximity to an urban area, or the length of time residing in a rural area. Overarching aspects of rural culture (i.e., stoicism, selfreliance, independence, reluctance to change) may not be universally shared or equally endorsed by all rural patients or their providers. Even so, rural communities can be insular and distrustful of outsiders, with many patients and providers preferring to work with people from their own rural community or at least the same state (27).

Although rural veterans are acknowledged as a special population with unique needs, demonstrated by national goals to increase outreach to veterans in rural areas, insufficient attention has been given to the ways in which rural culture might be influential at the site, provider, or patient level. Rural sites may have policies and procedures to accommodate a smaller or less specialized workforce (i.e., no specialty $\mathrm{MH}$ teams) or foster a personal relationship between providers and patients (i.e., preference for an inperson appointment before starting VTH). Providers in rural areas may be accustomed to operating independently, have minimal time or motivation to enact practice changes, and have important perspectives on how to address unique barriers to implementation faced by rural sites. Rural patients may wish to work with providers from their community who have firsthand knowledge of the local culture and customs. Developing a nuanced understanding of rurality enables us to understand and address rurality at multiple levels throughout the implementation process. Given these considerations, approaching rurality as a complex and multifaceted aspect of diversity is the cornerstone of our PIVOT-R approach for rural Veterans.

\section{Relationship building}

Building quality relationships is necessary for all successful implementation efforts, but relationships are even more important when working with rural sites. Leadership, providers, and staff at rural sites may be wary of outsiders' intentions due to exclusion from national efforts, discontinuation of rural initiatives, or isolation from professional colleagues. Rural site stakeholders may prefer to work with members of their community, amplifying the importance of early identification of internal facilitators as external facilitators may be perceived as evaluative outsiders rather than helpful partners. When entering a new rural site, external facilitators need to take time to foster collaborative and trusting relationships with stakeholders by inquiring about previous experiences with outside implementation and expectations of this partnership. External facilitators are typically located outside the implementation site, offering mentorship and a global perspective on the implementation process $(19,26)$. To maximize their credibility and expertise, ideal external facilitators should have implementation training, relevant specialized knowledge (i.e., innovation technology, target population), clinical or technical expertise, and dedicated time for implementation responsibilities.

\section{Engaging the site}

While PIVOT typically begins with a site visit, PIVOT-R encourages identification of an internal facilitator before a site visit with leadership. Developing a relationship with an internal facilitator who acts as a point of contact can improve understanding of the specific site context, demonstrate commitment to the site's priorities, and increase engagement by fostering trust and credibility. External facilitators outline the assistance they can offer and review success at other rural sites to begin crafting an implementation plan. Although ongoing evaluation of VTH growth is an important component of implementation, this process should be framed as one tool to gauge progress and reevaluate jointly identified VTH goals rather than a measure of a site's success or failure.

\section{Assessment of context and infrastructure}

PIVOT-R includes a comprehensive assessment of the rural context, available resources, and barriers to implementation. This assessment goes beyond the existing 
PIVOT implementation assessment, which was insufficient for understanding aspects of rurality, as it incorrectly assumed the existence of some resources. This early step is crucial because successful implementation of technologybased innovations, including VTH, requires significant infrastructure and effort from staff beyond clinical providers. PIVOT-R aims to identify limited resources or unexpected barriers that might otherwise be overlooked so that adaptations can be made.

Rural sites, particularly community clinics and surrounding areas, oftentimes have limited resources, which may include insufficient infrastructure (i.e., office space, high speed internet), equipment (i.e., webcams, dual monitors), or workforce development (i.e., specialty $\mathrm{MH}$ teams, training in evidence-based practices, continuing education opportunities, availability of colleagues). Limited resources may also include or contribute to high staff turnover and vacancies among leadership, frontline providers, and support staff, which can diminish the effectiveness of implementation efforts. Community clinics with a high proportion of unfilled positions or long-term vacancies may result in staff having multiple responsibilities/ roles or serving in "acting" or interim roles while leadership attempts to fill positions, as well as overall limited time to participate in the training necessary to begin using VTH. It can be unclear who is responsible for approving overall implementation efforts or completing specific tasks, and staff shortages can make cross training difficult. PIVOT-R applies experience and lessons learned to identify where to initiate implementation efforts, differentiate between necessary and desirable resources, and develop an action plan that reflects a site's existing resources.

\section{Balancing national expectations with site-level goals}

Implementing VTH at rural sites requires knowledge about national VHA expectations, including timelines for implementing innovations and related metrics, and their implications for rural sites. Decisions are often made at the national level to pilot innovations at large, urban medical institutions with the assumption that implementation efforts will trickle down to affiliated community clinics in rural areas. Unfortunately, rural sites may find that implementation strategies and lessons learned from urban sites are not applicable to their rural context and patients. The discrepancy between national VHA goals and rural site capabilities as well as lack of clarity about national expectations for rural sites can cause frustration and impede implementation among rural providers and patients. PIVOT-R relies upon external facilitators and their relationships with internal facilitators to monitor and communicate national metrics and timelines to ensure rural sites can apply that information to site-specific implementation efforts. The ongoing communication, transparency, and collaboration that PIVOT-R entails maximizes the likelihood of success.

\section{Case study: healthcare system serving predominantly rural veterans}

In May of 2018, we partnered with a VHA healthcare system in a Western mountain state to pilot our PIVOT-R approach. The healthcare system was selected for implementation because it had a high proportion of rural patients, no MH visits via VTH despite VTH approval since 2014, and a VA medical center with multiple affiliated community clinics to maximize the impact of implementation efforts. We applied lessons learned from PIVOT implementation evaluation in rural sites to pilot our PIVOT-R adaptations.

External facilitators began by identifying and building relationships with two internal facilitators, at different sites and in different disciplines, who could assist with orienting and introducing us to the health care system as we initiated implementation efforts at the main VA medical center. We began weekly virtual meetings with the internal facilitators focused on building rapport and hearing their reports of barriers and facilitators to implementation of VTH at their site. We then assessed the site's resources and collaboratively established goals. The internal facilitators' understanding of local dynamics helped us enter the system, enabling us to shift focus to building relationships, infrastructure, and resources to build trust and ensure early success. Taking time to assess the rural context and offering practical solutions enhanced our credibility with the internal facilitators resulting in introductions to and buy-in from clinic leadership and champion providers.

Although we communicated with on-site stakeholders as needed, we deliberately empowered the internal facilitators to be the local VTH experts as outlined in the initial PIVOT approach (21). Our individualized, concierge, and bottom-up approach to implementation was particularly effective at this rural site. We offered content and best practices, guidance about adapting to limited resources, and assistance navigating VTH processes. For example, we successfully helped local staff establish means to 
acquire telehealth equipment which addressed a significant barrier to VTH delivery. We also read and made sense of lengthy VTH memos and documents, frequently creating bulleted lists or 1-page summaries that highlighted relevant information, effectively addressing the notable time limitations previously reported by rural providers. In addition to support and trouble shooting from the external facilitators, we also trained the internal facilitators to be VTH experts capable of directly assisting and supporting their local colleagues. Bolstering the internal facilitators' expertise was especially effective for reaching providers who remained skeptical of working with external facilitators from outside their organization.

Approximately one year after partnering with the site and achieving some success with VTH implementation, the external and internal facilitators planned and executed an in-person site visit. Rural sites may not have the extensive organizational charts found at urban medical centers, but efforts should be made to include leadership and staff across levels (i.e., clinic, care line, site or medical center) and disciplines (i.e., psychology, psychiatry, social work, scheduling, telehealth). The internal facilitators identified and communicated with $\mathrm{MH}$ leadership to schedule the visit during the regular monthly $\mathrm{MH}$ all staff meeting. Being included on the meeting agenda demonstrated buyin from $\mathrm{MH}$ leadership, maximized provider attendance, and eliminated the need for providers to dedicate additional time to meet with us. The external facilitators met with providers, clinic leadership, schedulers, telehealth staff, and the Executive Leadership Team including the Medical Director for the healthcare system offering an overview of VTH technology and benefits, the PIVOT-R approach, national metrics, and site success. During the site visit we sought to "win hearts and minds" and demonstrate our dedication to the site's continued success. The health care system Director indicated strong support for VTH and our subsequent implementation efforts.

In September of 2019, the technology used to initiate VTH appointments/connections underwent a significant update and then changed to an entirely new system a short time later, causing widespread confusion and frustration. These changes threatened to undermine ongoing implementation efforts. Fortunately, the foundation previously developed with the internal facilitators and the connections established during the site visit helped us maintain the VTH gains that had been made and ultimately expand VTH delivery at this site. External facilitators also continued to attend national meetings, which provided early access to the most updated guidance and expectations, allowing us to communicate national VTH changes in real time to the rural site. Thus, the external facilitators proactively addressed and troubleshooted changes to VTH while working to support the internal facilitators and clinical champions. Throughout our collaboration, we witnessed firsthand how rural sites are sometimes excluded from national implementation efforts as they were among the last to receive telehealth system updates.

Implementation efforts at this site also contributed to changes in the PIVOT-R approach. Although we previously focused on the clinical aspects of VTH delivery, our experience demonstrated the importance of attending to the administrative side of VTH implementation. Effectively managing providers' schedules, specifically balancing in-person and VTH appointments, emerged as a crucial component of successful VTH implementation, particularly at rural sites with limited provider availability and increased travel time for patients. Designated VTH clinics need to be created before providers start using VTH delivery, and schedulers need the ability to "block" providers' inperson clinics to avoid double-booked appointments. When we learned that only a few schedulers could block providers' in-person clinics, we worked with internal facilitators and site leadership to ensure all schedulers were trained in VTH scheduling procedures and had blocking privileges. On a broader scale, we have recently focused on educating ourselves about scheduling procedures for VTH and identifying a Scheduling Champion at other implementation sites.

\section{Results}

\section{PIVOT-R case study evaluation}

As part of our continuous QI, we conducted both quantitative and qualitative data collection to evaluate the impact of PIVOT-R. We explored demographic data for the patients receiving VTH for $\mathrm{MH}$ in the healthcare system from FY2018 to FY2019 and compared the healthcare system with nationwide VHA rates on VTH use. We specifically examined the percentage change in number of veterans and providers using VTH for $\mathrm{MH}$ care at the implementation site. We also gathered feedback from rural providers and patients who used VTH for MH visits. This was designed as an FE with outcomes used to inform and modify the PIVOT-R approach throughout our implementation. 
Table 3 Demographic data for veterans receiving $\mathrm{MH}$ care via VTH at pilot site during FY2018-2019

\begin{tabular}{|c|c|}
\hline Variable & Number (\%) \\
\hline \multicolumn{2}{|l|}{ Rurality } \\
\hline Rural & 84 \\
\hline Urban & 16 \\
\hline \multicolumn{2}{|l|}{ Gender } \\
\hline Female & 37 \\
\hline Male & 63 \\
\hline \multicolumn{2}{|l|}{ Age, years } \\
\hline 20-29 & 4 \\
\hline 30-39 & 31 \\
\hline $40-49$ & 19 \\
\hline $50-59$ & 18 \\
\hline $60-69$ & 15 \\
\hline $70-79$ & 11 \\
\hline 80-89 & 2 \\
\hline \multicolumn{2}{|l|}{ SVC } \\
\hline $0 \%$ & 1 \\
\hline $10-20 \%$ & 12 \\
\hline $30-60 \%$ & 21 \\
\hline $70-100 \%$ & 48 \\
\hline Missing/NSC & 18 \\
\hline \multicolumn{2}{|l|}{ OEF/OIF } \\
\hline Yes & 25 \\
\hline No/unknown & 75 \\
\hline
\end{tabular}

$\mathrm{MH}$, mental health; VTH, video telehealth to home; NSC, nonservice connected; OEF, Operation Enduring Freedom; OIF, Operation Iraqi Freedom; SVC, service connected.

\section{Quantitative data}

All data reported are for the specific healthcare system where we used PIVOT-R to implement VTH; explicit comparisons to national VHA data are made to highlight the effectiveness of our PIVOT-R strategy. Descriptive analyses of data indicate PIVOT-R effectively increased the uptake of VTH for MH care at the healthcare system. Of all veterans receiving MH care in FY2018 and FY2019, $69 \%$ were rural. However, of the veterans using VTH for MH care, 84\% were rural. Similarly, in FY2018 and

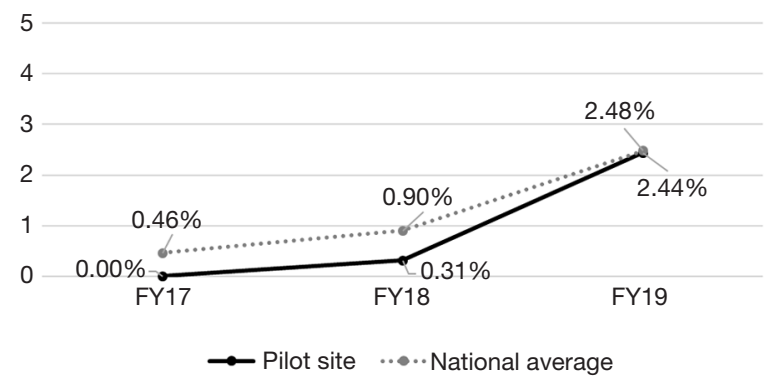

Figure 1 Percentage of veterans receiving $\mathrm{MH}$ services via VTH from FY17-FY19. MH, mental health; VTH, video telehealth to home.

FY2019 13\% of all veterans receiving $\mathrm{MH}$ care were women; but $38 \%$ of veterans using VTH for $\mathrm{MH}$ care were women (see Table 3 for demographic data). In FY2019, the percentage of veterans receiving $\mathrm{MH}$ care via VTH at the healthcare system was 10 times greater than it was in FY2018, compared to a 3.5 times increase between FY2018 and FY2019 in the mean percentage of veterans receiving MH care via VTH nationally (see Figure 1). Importantly, by FY2019 the percentage of veterans receiving $\mathrm{MH}$ care via VTH at the healthcare system matched the mean nationwide VHA percentage. The percentage of $\mathrm{MH}$ providers using VTH increased to $43.24 \%$ by the end of FY2019 (see Figure 2).

\section{Rural provider feedback}

Feedback from rural providers emphasized VTH's ability to improve access to care for rural patients and areas with limited $\mathrm{MH}$ providers. Although rural providers reported more problems with technology and connectivity than their urban colleagues, likely related to limited cellular or internet infrastructure and mountainous geography in the region, they stressed that the benefits far outweighed the challenges. Rural providers elaborated on how effective VTH was for connecting rural patients who would otherwise not receive care due to travel time to clinic, anxiety, co-morbid medical issues, or being out of state for work or pleasure. One provider described using VTH for couples counseling, while one member of the couple traveled for work which she believed ultimately saved their marriage; without VTH, couples counseling would have been delayed for several months. Rural providers also described a variety of ways they had introduced VTH to 
100

80

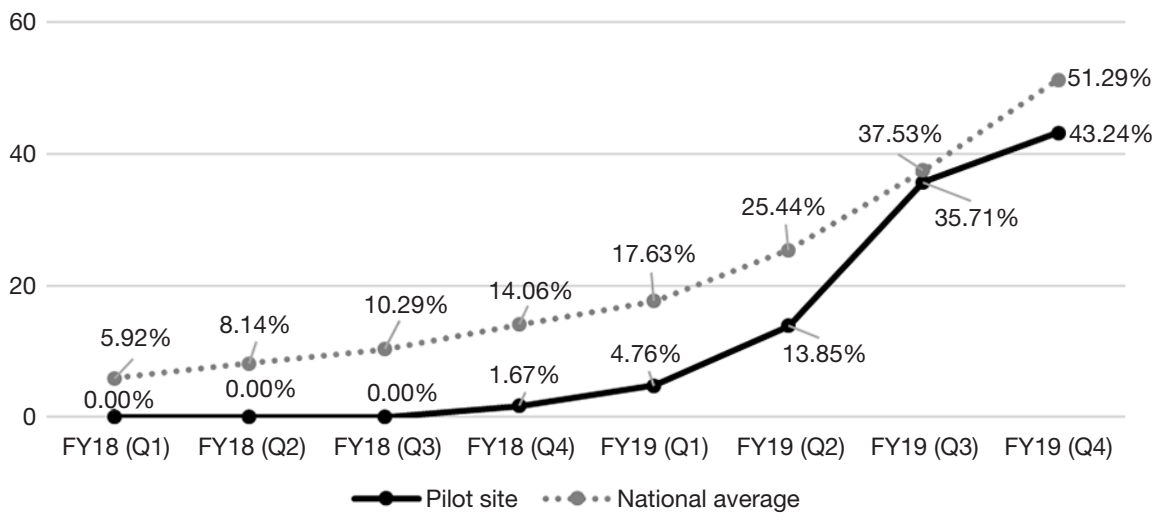

Figure 2 Percentage of MH providers delivering services via VTH from FY18-FY19. MH, mental health; VTH, video telehealth to home.

their rural patients, usually emphasizing convenience and ease of use.

\section{Rural veteran feedback}

Rural veterans described how VTH helped them engage in $\mathrm{MH}$ care, increased their quality of life, and improved perceptions of the VA despite challenges with technology. Interestingly, not all veterans living in a rural-categorized area self-identified as rural, contrasting their location with more isolated or rural surrounding areas and citing proximity to an urban area (e.g., 20 minutes from town). While a rural label was not uniformly endorsed, veterans consistently described the relative lack of resources (i.e., shopping, medical care) that exists throughout the state. They discussed how even urban areas in their state have limited MH providers, making VTH especially effective for receiving $\mathrm{MH}$ care. Veterans reported that VTH allowed for more frequent visits (i.e., weekly) and decreased the impact of staff turnover and vacancies, as veterans could receive care from providers at other sites within the healthcare system during transition times. Rural veterans also unanimously indicated willingness to use VTH for $\mathrm{MH}$ care in the future with some wishing that VTH was more widely available. One veteran described how VTH was a "good tool" for their largely rural state because there are areas along the Canadian border with no $\mathrm{MH}$ access for hundreds of miles.

\section{Unique factors for VTH in rural communities}

The FE of PIVOT-R in this highly rural health care system revealed some unique considerations for implementation of VTH in rural areas. Feedback from rural providers highlighted how VTH might help address the unique challenge of rural patients with a history of violence. Within VHA, some patients with a behavior flag in their chart are required to have a police escort when accessing in-person MH care. This can create additional barriers for rural patients because some highly rural states or health care systems only have one location with police, greatly increasing travel time/distance for the patient and travel pay/overnight accommodations/meals reimbursement for the site. Inperson, home-based care is sometimes used but involves provider travel time/cost and potential safety risks (28). With the development of site-specific standard operating procedures and clinical guidelines, VTH may offer a possible solution for these patients to receive $\mathrm{MH}$ care.

Our experience using PIVOT-R reiterated the necessity of VTH emergency procedures that reflect each specific site's resources (29). When working with urban patients, providers may readily utilize local emergency resources 
to conduct a wellness check when concerned about the well-being of a patient. However, caution is advised when sending police to a rural patient's home unannounced due to a higher presence of firearms and skepticism about law enforcement. This knowledge of rural communities emphasizes the importance of collaborating with rural providers, stakeholders, and police to develop feasible site-specific $\mathrm{MH}$ emergency procedures, communicating clearly with rural patients about VTH safety protocols, and partnering closely with local law enforcement.

\section{Discussion}

PIVOT-R integrates existing knowledge of rurality with lessons learned from previous VTH implementation efforts at rural sites, emphasizing rurality as an important diversity factor impacting the uptake of VTH among rural providers and patients. The inclusion of a comprehensive assessment of the rural site, including infrastructure and resources, greatly improves understanding of a site's specific needs and enables a tailored approach that targets relevant barriers. PIVOT-R prioritizes relationships between external and internal facilitators, with internal facilitators emerging as a critical component for success due to their role in engaging stakeholders and navigating the rural site.

Evaluation of our PIVOT-R approach with one highly rural health care system suggests its potential for increasing VTH uptake at other rural sites. Our implementation efforts from FY2018 to FY2019 moved the site from no completed VTH visits to successful integration of VTH for $\mathrm{MH}$, with both veterans and providers, throughout the health care system.

\section{Future directions}

Our experience using PIVOT-R to implement VTH in a highly rural health care system reinforced the value of telehealth technology as an important resource for rural sites. While the telehealth technology has understandably focused on delivery of clinical care, including both clinical and non-clinical applications in future implementation efforts could maximize the effectiveness for rural sites.

\section{VTH in rural areas}

Our understanding of rural communities, patients, and providers was greatly improved by our experience piloting PIVOT-R in a highly rural health care system and highlighted unique aspects for consideration moving forward. It is important to continue examining rurality as a cultural factor at additional sites, specifically noting any regional or geographic differences. Additionally, little is known about the intersection of rurality with other aspects of diversity (i.e., race, gender, disability) and its impact on the uptake of VTH. As VTH continues to expand, understanding the influence of intersecting identities will enable improved marketing to maximize the benefit of VTH for underserved populations.

PIVOT-R was piloted within the VA system where all VTH delivery is covered under federal supremacy and "Anywhere to Anywhere" legislation which allows the provision of $\mathrm{MH}$ care throughout the United States. Thus, veterans who are enrolled in VA and engaged in $\mathrm{MH}$ care can continue to meet with their provider, even when located in another state for work or personal reasons. The approval of "Anywhere to Anywhere" simplified implementation and maximized the impact of VTH delivery for rural veterans, especially in northern states, where many residents choose to move south for the winter. Future efforts could expand education about VTH and "Anywhere to Anywhere" for both providers and Veterans, including patient-facing marketing, to increase awareness of VTH as an option for MH care.

Currently, telehealth guidelines in the public sector do not universally allow for provision of care across state lines, overwhelmingly limiting VTH delivery in the private sector to within state care. Even with in state restrictions, VTH can increase access to $\mathrm{MH}$ care in rural areas by enabling patients to receive care at home or another convenient location. It remains to be seen when and how state licensing boards and laws will evolve to reflect the possibilities that telehealth technology presents, with $\mathrm{MH}$ providers having an important role in informing and shaping future guidelines.

\section{Beyond clinical care}

Telehealth technology has advanced rapidly in recent years which has made it more user friendly and created new opportunities for non-clinical applications. Providers in rural areas oftentimes have less access to training, supervision, and peer consultation within and across disciplines than their urban colleagues, and telehealth technology is ideally suited to address those gaps. As sites and providers become increasingly comfortable with VTH technology, that same technology can be extended for 
EBP trainings, tele-consultation, and care coordination. Our team has already successfully leveraged telehealth technology to conduct virtual implementation, VTH practice calls, and implementation meetings with internal facilitators and clinical champions. Using telehealth technology beyond clinical care can improve confidence with technology, connection with colleagues, and coordination or continuity of care which could positively impact job satisfaction and professional development.

Telehealth technology can similarly be expanded to improve engagement with rural veterans' caregivers and/or family members. VTH is well-suited to provide psychoeducation, conduct care planning meetings, streamline scheduling, offer support, and collect collateral information. When rural veterans' family members are in geographically distant locations, it can be difficult to include them in treatment planning, contributing to a patient's isolation. Even when family members or caregivers are nearby, VTH minimizes costs (i.e., travel time and distance, time away from work or family responsibilities) to a patient's support network. By enabling a connection with a provider from any convenient private location (i.e., their home in another state, work), VTH can decrease the burden and stress on a rural veteran's support system.

\section{Acknowledgments}

The authors would like to thank Byron Bair, MD, and Nancy Dailey, MSN, RN, for project guidance and mentoring.

Funding: This work supported by a grant from the VA Office of Rural Health, Veterans Rural Health Resource Center-Salt Lake City and partly supported by the use of facilities and resources of the Houston VA HSR\&D Center for Innovations in Quality, Effectiveness and Safety (grant number CIN13-413) and the VA South Central Mental Illness Research, Education and Clinical Center. The opinions expressed are those of the authors and not necessarily those of the Department of Veterans Affairs, the US government, or Baylor College of Medicine.

\section{Footnote}

Conflicts of Interest: All authors have completed the ICMJE uniform disclosure form (available at http://dx.doi. org/10.21037/mhealth.2020.03.02). The authors have no conflicts of interest to declare.
Ethical Statement: The authors are accountable for all aspects of the work in ensuring that questions related to the accuracy or integrity of any part of the work are appropriately investigated and resolved. This study was designated as a quality-improvement project and did not require IRB approval.

Open Access Statement: This is an Open Access article distributed in accordance with the Creative Commons Attribution-NonCommercial-NoDerivs 4.0 International License (CC BY-NC-ND 4.0), which permits the noncommercial replication and distribution of the article with the strict proviso that no changes or edits are made and the original work is properly cited (including links to both the formal publication through the relevant DOI and the license). See: https://creativecommons.org/licenses/by-nc-nd/4.0/.

\section{References}

1. Association NRH. Rural Health Voices. 2020. Available online: https://www.ruralhealthweb.org/blogs/ ruralhealthvoices/march-2018/essential-outreach-expandingmental-health-suppor. Accessed January 10, 2020.

2. Hoeft TJ, Fortney JC, Patel V, et al. Task-sharing approaches to improve mental health care in rural and other low-resource settings: A systematic review. J Rural Health 2018;34:48-62.

3. Hughes MC, Gorman JM, Ren Y, et al. Increasing access to rural mental health care using hybrid care that includes telepsychiatry. Rural Ment Health 2019;43:30-7.

4. Andrilla CHA, Patterson DG, Garberson LA, et al. Geographic variation in the supply of selected behavioral health providers. Am J Prev Med 2018;54:S199-207.

5. Jacobs JC, Blonigen DM, Kimerling R, et al. Increasing mental health care access, continuity, and efficiency for veterans through telehealth with video tablets. Psychiatr Serv 2019;70:976-82.

6. Fischer EP, McSweeney, JC, Wright P, et al. Overcoming barriers to sustained engagement in mental health care: Perspectives of rural veterans and providers. J Rural Health 2016;32:429-38.

7. Thorne KL, Price E, Fiske A, et al. Traumatic stress and suicide risk: the role of rural origin. Rural Ment Health 2017;41:42-53.

8. Teich J, Ali MM, Lynch S et al. Utilization of mental health services by veterans living in rural areas. J Rural Health 2017;33:297-304. 
9. Stetler CB, Legro MW, Rycroft-Malone J, et al. Role of external facilitation in implementation of research findings. A qualitative evaluation of facilitation experiences in the Veterans Health Adminisytration. Implement Sci 2006; 1:23.

10. Office of Mental Health and Suicide Prevention. 2019 National Suicide Prevention Annual Report. Department of Veterans Affairs; 2019.

11. Morland LA, Mackinosh MA, Glassman LH, et al. Homebased delivery of variable length prolonged exposure therapy: A comparison of clinical efficacy between service modalities. Depress Anxiety 2020;37:346-55.

12. Fletcher TL, Hogan JB, Keegan F, et al. Recent advancs in delivering mental health treatment via video to home. Curr Psychiatry Rep 2018;20:56.

13. Kruse CS, Krowski N, Rodriguez B, et al. Telehealth and patient satisfaction: A systematic review and narratifew. BMJ Open 2017;7:e016242.

14. Boykin DM, Keegan F, Thompson KE, et al. Video to home delivery of evidence-based psychotherapy to veterans with posttraumatic stress disorder. Front Psychiatry 2019;10:893.

15. Russo JE, Mccool RR, Davies L. VA telemedicine: An analysis of cost and time savings. Telemed J E Health 2016;22:209-15.

16. Health Benefits. US Department of Veterans Affairs. Available online: https://www.va.gov/ HEALTHBENEFITS/vtp/beneficiary_travel.asp. Accessed January 10, 2020.

17. Office of Connected Core Quality and Training Division. Powering World-Class Virtual Care for Veterans - Annual Report: US Department of Veterans Affairs, September 2019.

18. Connolly SL, Miller CJ, Lindsay JA, et al. A systematic review of providers' attitudes toward telemental health via videoconferencing. Clin Psychol Sci Pract 2020. [Epub ahead of print].

19. Hogan J, Boykin, D, Schneck C, et al. Clinical lesson from virtual house calls in mental health: The doctor is in the house. Psychiatr Clin North Am 2019;42:575-86.

20. McCann RA, Lingam HA, Felker BL, et al. Practical and regulatory considerations of teleprescribing via CVT. Curr Psychiatry Rep 2019;21:122.

21. Lindsay JA, Day SC, Amspoker AB, et al. Personalized implementation of video telehealth. Psychiatr Clin North Am 2019;42:563-74.

22. Lindsay JA, Hudson S, Martin L, et al. Implementing video to home to increase access to evidence-based psychotherapy for rural veterans. J Technol Behav Sci 2017;2:140-8.

23. Ellimoottil C, An L, Moyer M, et al. Challenges and opportunities faced by large health systems implementing telehealth. Health Aff (Millwood) 2018;37:1955-9.

24. Kane CK, Gillis K. The use of telemedicine by physicians: Still the exception rather than the rule. Health Aff (Millwood) 2018;37:1923-30.

25. Stetler CB, Legro MW, Bowman C, et al. The role of formative evaluation in implementation research and the QUERI experience. J Gen Intern Med. 2006;21:S1-8.

26. Ritchie MJ, Dollar KM, Miller Chj, et al. Implementation Facilitation Training Manual. Using Implementation Facilitation to Improve Care in the Veterans Health Administration (Version 2): Quality Enhancement Research Initiative (QUERI) for Team-based Behavioral Health. Veterans Health Administration; 2017.

27. Cheesmond NE, Davies K, Inder KJ. Exploring the role of rurality and rural identity in mental health help-seeking behavior. J Rural Ment Health 2019;43:45-59.

28. Semeah LM, Campbell CL, Cowper DC, et al. Serving our homeless veterans: Patient perpetrated violence as a barrier to health care access. Journal of Public and Nonprofit Affairs 2017;3:223-34.

29. McGinn MM, Roussev MS, Shearer EM, et al. Recommendations for using clinical video telehealth with patients at high risk for suicide. Psychiatr Clin North Am 2019;42:587-95. doi: $10.21037 /$ mhealth.2020.03.02

Cite this article as: Day SC, Day G, Keller M, Touchett H, Amspoker AB, Martin L, Lindsay JA. Personalized implementation of video telehealth for rural veterans (PIVOT-R). mHealth 2021;7:24. 